Shamu, S. et al. (2013). Opportunities and obstacles to screening pregnant women for intimate partner violence during antenatal care in Zimbabwe. Culture, Health \& Sexuality: An International Journal for Research, Intervention and Care, 15(5): 511-524 http://dx.doi.org/10.1080/13691058.2012.759393

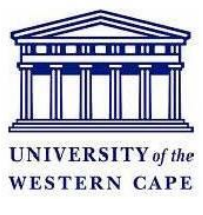

\title{
Opportunities and obstacles to screening pregnant women for intimate partner violence during antenatal care in Zimbabwe
}

Simukai Shamu, Naeemah Abrahams, Marleen Temmerman and Christina Zarowsky

\begin{abstract}
Pregnancy offers an opportunity for midwives to recognise and respond to women experiencing intimate partner violence (IPV). However, most antenatal care interventions have been conducted in private specialist services in high-income countries and do not address the structural and cultural realities of developing country settings. We report on an exploratory qualitative study conducted in antenatal public health facilities in Harare, Zimbabwe, involving six in-depth interviews with midwives and seven FGDs with 64 pregnant and postpartum women. Recorded interviews were transcribed verbatim and analysed using thematic content analysis. We found that identifying and responding to IPV in antenatal care is hampered by inadequate human, financial and infrastructural resources as well as poor support of gender-based violence training for midwives. Midwives had divergent views of their role, with some perceiving IPV as a non-clinical, social and domestic problem that does not require their attention, while others who had been sensitised to the problem felt that it could easily overwhelm them. A comprehensive response to IPV by midwives would be difficult to achieve in this setting but sensitised midwives could respond to cues to violence and ultimately assist abused women in culturally sensitive and appropriate ways.
\end{abstract}

\section{Introduction}

Intimate partner violence (IPV) during pregnancy impacts negatively on women's reproductive health and on their babies. Studies have shown its association with unsafe abortion, gynaecological disorders, pregnancy complications, miscarriage, low birth weight and STI/HIV infection (Audi et al. 2008; Heise, Ellsberg, and Gottmoeller 2002; Silverman et al. 2006; WHO 2005). Pregnancy offers a unique opportunity to identify and assist women experiencing IPV (Bacchus et al. 2004) as many pregnant women visit antenatal care clinics repeatedly. Recent reviews have also shown that identification of abuse increases sharply when universal routine screening is conducted in health settings like antenatal care (Bacchus et al. 2010; O'Campo et al. 2011). Literature on patterns of IPV suggests that violence can begin, continue or increase during pregnancy (Taillieu and Brownridge 2010), pointing to the important role that screening and the provision of comprehensive care to 
abused women could play in decreasing the impact of abuse on the health of women and their children (O'Campo et al. 2011). This is particularly relevant for countries and regions where high levels of IPV have been reported. The prevalence of physical IPV during pregnancy (8\%) in Zimbabwe (CSO and Macro 2007) is among the higher levels recorded recently (Shamu et al. 2011).

A recent review of the effectiveness of universal and routine IPV screening in health settings concluded that a multiple-component programme that includes initial and ongoing staff training, effective screening protocols, institutional support, and immediate onsite or offsite referral services increases disclosure and identification of abused women (O'Campo et al. 2011). Although routine screening for violence in health settings has been endorsed by many health professional organisations, including the US academies and colleges of gynaecologists and paediatricians (Roelens et al. 2006; Waalen et al. 2000), few health settings in the world have begun implementing this intervention. Most African health settings do not meet the above criteria for comprehensive programmes to respond to IPV because of their weak health systems, lack of infrastructure and human resources as well as cultural reasons that inhibit discussing or disclosing domestic life outside of the home. Unlike Western countries, many health systems in Africa are still to recognise IPV as a health problem or that the health sector has a role to play. The fact that any screening programme must lead to appropriate care imposes major challenges to ill-equipped and short-staffed health and social care systems in many environments in Africa. However, empirical assessments of the 'readiness' of African healthcare settings for comprehensive IPV interventions are generally lacking.

Women's views about being screened in health settings have been documented as generally pro-screening (Ramsay et al. 2002). In all, 98\% of the 1313 rural and urban female patients interviewed in Australia, believed it was a good idea to be screened (Webster et al. 2001). In the USA, abused women were one and a half times more likely to agree to screening than women who reported no abuse (Gielen et al. 2000).

Bacchus, Mezey, and Bewley's (2002) qualitative study in the UK showed that women were willing to participate in IPV interventions if their safety and confidentiality were guaranteed. Women preferred to be interviewed by trained health professionals who were empathetic, non-judgemental and genuinely interested in the client's health and wellbeing. The importance of cultural sensitivity in screening to encourage disclosure of violence has been highlighted (Hindin 2006) and understanding the client's language and nonverbal cues when trying to identify and respond to IPV is essential for its success. A trusting relationship between a midwife and a client helps when responding to violence (Stenson, Sidenvall, and Heimer 2005). Although these studies demonstrate women's positive attitude to being screened and how culture influences responses to IPV, they were all conducted in developed countries and predominantly in specialist, private, obstetrical- gynaecological offices rather than in busy public primary health-care settings. Similar studies about the views of pregnant women in African health care settings are only beginning (Joyner and Mash 2012; Undie et al. 2012). In Zimbabwe and other 
developing settings, where most pregnant women are attended to by nurses in public health settings (CSO and Macro 2007), women's views about being screened for IPV could be different.

Screening for IPV is not part of current clinical practice in Zimbabwe, yet high IPV rates during pregnancy in Zimbabwe (CSO and Macro 2007) seem to warrant it. This paper aims to explore the current environment in order to identify opportunities and obstacles for interventions aimed at identifying and responding to IPV in antenatal care. The paper presents formative research from a broader study of the dynamics of IPV during pregnancy in Zimbabwe. The formative study provided an opportunity to explore perceptions and experiences of nurse midwives working in Zimbabwe's public maternity services regarding IPV among pregnant women, including possible responses in the clinic setting. Perceptions and experiences of women attending these services were also explored.

\section{Methods \\ Study setting}

The setting of the study is within six public antenatal care clinics located in lowincome residential areas in Harare. These clinics were purposively selected because of their long- standing relationship with a local university teaching programme. Most clinics included in the study had antenatal services three days a week and up to 35 women were attended each day per clinic. Clinics controlled the maximum number of pregnant women by dismissing 'excesses' if the turn up was higher than their daily quota. There were chronic staff shortages in the maternity services owing to brain drain and nurses would often be shifted from one department to another. Each antenatal clinic was located in a polyclinic, which also housed the primary health care and family health clinics. Women gave birth in the maternity ward of the antenatal care clinic and brought their babies to the family health clinics. At the time of the study, a fee of US\$50 was charged to each woman for antenatal, labour and post-natal care.

We sought and obtained ethical clearance from the Medical Research Council of Zimbabwe and the University of the Western Cape Senate Research committee, whilst permission to conduct the study at the six facilities was provided by the City Health Directorate. To preserve the anonymity of women, health workers and clinic sites, we have replaced their names with pseudonyms.

\section{Design}

We used qualitative methods, including in-depth interviews, FGDs and observation. The in-depth interviews were held with service providers, and FGDs were held with pregnant and nursing women to explore health workers' and women's views and perceptions of responding to IPV (physical, sexual and emotional) during pregnancy. During the data collection phase, the researcher spent time in the clinic and observed the clinic space and clinical interactions during the antenatal care sessions. 


\section{Interviewing health workers}

At each of the six clinics, one senior nurse midwife (sister-in-charge) in charge of the maternity clinic was purposively selected to participate in the interviews (six in-depth interviews). The ages of midwives ranged between 40 and 60 years. The first author made appointments with midwives, sought and received written informed consent and held in- depth key informant interviews privately in their offices. The interview guide explored how midwives recognised IPV, how they dealt with suspected or identified cases, what obstacles they faced in trying to recognise abused women and opportunities available to them and the health system to help abused women. Interviews were held in English but participants could switch to vernacular (Shona), especially when quoting women's reported experiences. The interviews were digitally recorded, supported by written notes after each interview to complement the interview information, which was transcribed in preparation for data management and analysis.

\section{Focus-group discussions with pregnant women}

The researcher approached women at the six clinics where they queued to receive antenatal or postnatal care services and informed them about the study. We invited all women in the queue if they were less than 10 or randomly invited up to 10 if more. Consent procedures followed for those who accepted the invitation. The first author led the discussions in the vernacular and a female research assistant operated the digital recording and took field notes, which contributed to the data. The primary goal of the discussions was to explore experiences of IPV during pregnancy and to help develop a tool for the broader quantitative study. Information about how health providers respond to IPV during pregnancy emerged during the discussions. A total of 64 women participated in seven FGDs held separately with pregnant and nursing mothers at the six health facilities.

\section{Data analysis}

We transcribed the digitally recorded data verbatim and parts of the transcriptions that were in Shona were translated into English. An independent translator doublechecked the translations by listening to audio-records and back translated the sections of the transcript that were originally captured in Shona back into Shona for accuracy and consistency. The first author repeatedly read the transcripts and constructed codes in line with research objectives and the co-authors and additional researchers independently reviewed sections of transcripts and commented on the interim analysis. We used Open Code qualitative software to organise the data into codes and categories. Common themes from the interviews were identified and data were organised into categories. New codes were formulated as themes continued to emerge during the process of re-reading the scripts. Thematic content analysis was employed to systematically analyse the content of each theme.

\section{Findings}

Four core themes related to the possibilities of screening and/or intervening against IPV in these settings were identified: identification of abused women by midwives, women's accounts of midwives' interpretation of IPV, midwives' experiences of responding to IPV in a planned intervention in antenatal care, including how this 
opportunity was lost, and the influence of culture and the law in identifying and responding to abused women.

\section{Identifying abused women: current practice}

All midwives stated that they had no specific training, skills or competence to recognise abused women during antenatal and postnatal care and that no facilities were conducting any form of screening for IPV. Midwives reported diverse ways of responding to IPV. They reported that they were able to recognise only the more obvious cases such as those who had bruises or injuries on their bodies. They indicated that physical violence was a bit easier to detect compared to other forms of violence: 'Perhaps if there are quite obvious marks from battering such as some bruises' (Anna, midwife, Mutenda Clinic). Visible emotions were also recognised as signs of problems in intimate relations as described, 'And most of the times you will see this by crying' (Fadzai, midwife, Chineka Clinic).

Midwives mentioned that they mainly relied on mothers' willingness to reveal their experiences of violence, which mothers rarely did. They did not see it as their role to identify the violence: 'Usually it is the mother who comes out if she has problems .. . We do not screen ... It is for the mother to come out and say 1 have a problem at individual level' (Bridget, midwife, Nekanda Clinic). Midwives recognised that women also do not talk about the abuse easily and that the violence is revealed in indirect ways such as when condom use was discussed with the women. As one midwife remarked:

'They do not come in the open when it comes to the issue of sex after HIV tests. They take condoms in fear because they say, 'I will be beaten up at home if he finds condoms in my bag'. They want to consult with partners first before taking condoms. Their partners accuse them of prostitution if they find them with condoms' (Carol, midwife, Bungu clinic).

Midwives reported that very few women spontaneously disclosed their experiences. One midwife described how often she identified abused women in the following manner, 'Very rare. I don't want to lie, very, very rare' (Bridget, midwife, Nekanda Clinic). Although the midwives assigned responsibility to women to disclose they also recognised their own role and the dynamics of the provider-client relationship:

'No I haven't come across such cases [sexual and emotional violence]. Maybe they are not putting it across clearly and I think with the time that we have we are not probing enough as well. So we treat maybe on the surface and some of these issues go unnoticed' (Anna, midwife, Mutenda clinic).

This is further complicated by a lack of technical or professional language for dealing with IPV as a health issue. Women tend to use non-direct language when describing partner and sexual violence and midwives perceived and experienced difficulties in responding to such a sensitive phenomenon in vernacular language during history taking. One midwife at Vurinda Clinic remarked that 'It is difficult to ask in Shona if they are experiencing partner or family abuse'. 
At one of the busiest antenatal care clinics (Madzive clinic), the midwife mentioned that they were not asking violence questions because they thought the prevalence and effects of violence during pregnancy were insignificant and did not require them to intervene:

'You know why I am a bit hesitant? It's because usually what leads us to investigate is the frequency of the patients complaining about that. When we don't get the complaints usually we don't want to get involved ... the frequency and occurrence of the problem, the magnitude of the problem. [When] we are worried about that ... [we] try to investigate. Currently to be honest l haven't had a case of a woman being mistreated by a husband...' (Diana, midwife, Madzive clinic).

However, research suggests that most violence victims do not report their experiences without being asked (Roelens et al. 2006) and, as noted above, midwives indicated that they recognised that violence could be concealed. Midwives also recognised that one needed a great deal of time and skill to identify a case. One midwife gained some sensitisation and experience by witnessing a domestic violence non-governmental organisation intervention at their clinic a few years ago. The exposure to this programme in the clinic has made her view things differently and she consistently showed much more empathy than the other midwives and realised that listening to patients was critical. This she demonstrated when she said mothers only open up after a great deal of time and effort:

'There are cases when someone would come with a queer complaint. It won't be looking like it's the case. You will see that this person is not sick but there is something wrong. Then you sit and discuss with that person that's when she will open up. She will [then] say nurse I am not feeling well because my husband is doing this and this. They open up that way.' (Fadzai, midwife, Chineka Clinic)

Midwives at Chineka clinic reported how suspicion of abuse was raised in the labour ward when the women's social problems with their partners were recognised. An example was given when a midwife explained how a woman did not have the prepared items that the father normally buys in preparation for the baby as nonbuying of these items was demonstration of father's non-interest in the baby. She reported that they did not think it was safe for a woman to be discharged home after the birth of her baby and had kept her at the clinic for some time until they were satisfied that she had some support from family.

'So sometimes you end up saying to her you are not going home. You don't discharge her. You keep her there. Then when people come to visit her you try to find out if there is the mother. Or even someone who is very close to her. You then try to find out if there are problems at home. If she is facing any problems. Maybe .. . if she is having any problems with the husband' (Fadzai, midwife, Chineka Clinic).

This response to ensure women's safety is evidence of midwives' own initiative despite working in a system that does not encourage them to do so. 
The interviews reflect midwives' mixed feelings about responding to IPV in antenatal care and suggest an unresolved tension between what is considered an appropriate professional response versus a private matter to which they as women felt obliged to respond. The majority strongly viewed it as adding another huge task to a skeletal staff at the facilities, despite also viewing it as important for pregnant mothers. One respondent reported: 'Do you want to add more work to us? We treat patients and you want to involve us in those who are battered? It's a lot of work, though good.' (Ednah, midwife, Vurinda clinic). Midwives drew a line between their clinical work for which they trained and what they called social problems, including violence, which they did not think should be in their clinical practice. The amount of time required to assist women experiencing violence during the clinic sessions could be viewed as a constraint should IPV screening be implemented in antenatal care. A midwife who learnt that more time is needed to deal with women in abusive relations, which is not possible with the long queues during working hours at the clinic, said that talking to women outside of the clinic setting could be easier:

'Some of them they don't disclose. ... If you take time with that mother that's when you find out there is something wrong. That's when she will open up. Personally, I live right here. There are some whom I see and they open up. Such that after being tested [for HIV] they come to me. She might not come to the clinic. She will come to my house ... . and then we sit down and discuss. I tell her the options and what she can do. When you are doing it out there they will be seeing it ... differently ... we will be talking about it at the same level at home ... or some of them at church ...' (Fadzai, midwife, Chineka Clinic)

\section{'Nurses taught us not to refuse our husbands sex'}

Providing health information and advice (which include nutrition, hygiene, physical exercises and sexual health education) during group or one-to-one sessions was an important aspect of education during antenatal care visits. Women's experiences of these teaching moments revealed health workers' poor recognition of intimate partner sexual violence. Negative gender stereotypes were inadvertently reinforced by midwives. While pregnant women expected to receive information on how to avoid violence in their relationships, they were advised and taught how to subordinate to their male partners and accept forced sex. As reported during FGDs with women:

'We came yesterday and the nurses taught us not to refuse our husbands sex because they will go out to small houses [girlfriends]. Even when you feel you don't like it [sex] just do what you can so that you keep him satisfied. Try to push until labour. These are some of the teachings that you will not be aware of. They [midwives] said breathe with two entrances [orifices] [Laughter]. Some [women] say 'at six months l will no longer have sex'. Do not be fooled just try and give him sex so that he will be satisfied" (Doreen, FGD with pregnant women, Madzive Clinic).

Although this teaching was intended to reduce the risk of HIV infection, women were given the responsibility to ensure their male partners do not engage in multiple 
concurrentsexual relationships. These recommendations from the midwives taught women that male sexual needs superseded their own sexual and psychological needs and the health of their unborn child. That such advice is at least sometimes taken to heart was revealed by one woman who said 'As for me when 1 came from the clinic 1 changed at once. I am now doing what $\mathrm{l}$ can and not to deny him [sex]' (Patricia, FGD with pregnant women, Madzive clinic).

However, whilst many women in the group discussion seemed to have accepted the advice, a few women rejected this subjugation and even reversed the teachings completely as shown below:

'As for me 1 even lied when 1 came back from clinic. I saw that I was going to die [Laughter]. I told him that the nurses said 'you should not abuse and force me to have sex'. If you force me, 1 will go back and report you to the nurses and you will go to the police. If you are taken by the police what will 1 do'. He understood me" (Makanaka, FGD with pregnant women, Madzive Clinic)

The data from the FGD with women are supported by the interviews with midwives. Diana, a midwife at Madzive Clinic reported that they advised women not to refuse sex during pregnancy as their clinical practice does not forbid sex during pregnancy.

The poor knowledge and understanding by health workers of what constitutes sexual violence clearly impedes their ability to recognise IPV. If midwives endorse some forms of sexual violence they are also unlikely to recognise other forms, even if women's statements provide cues to experiences of sexual violence. The lack of knowledge on the part of midwives is indicated in the following quote:

'With pregnant mothers, at times we wouldn't even know that this [forced sex] is abuse because when we meet them they report [refusing sex] ... we wouldn't be able to know whether this person is being abused or not ... When we ask them they say 'the stomach is now too big and I don't want anybody on top of it' (Fadzai, midwife, Chineka Clinic)

\section{Dealing with cases: current practice}

In many cases, health workers did not take any action once they became aware of cases of violence other than just noting them as social problems. Some cases were reported to a referral (tertiary) hospital. External bodily injuries were reported to the police for prosecution and to the doctor for proper assessments. Musasa, a nongovernmental organisation that works against gender-based violence in Harare, was also mentioned as a referral centre for abused women. As one midwife remarked, 'If it is a serious case we can refer them to places like Musasa but we have never referred anybody' (Bridget, midwife, Nekanda clinic). At Mutenda clinic, there used to be a non-governmental organisation that employed midwives and counsellors trained in IPV counselling. They holistically helped abused women by counselling them and providing financial resources and other support needed. Some midwives remarked that since it was a matter of domestic issues, it was proper to refer such issues to 
traditional or family courts as per their culture. The midwife at Nekanda clinic reported, 'We usually advise them to use the support systems in their homes- aunties, grandmothers'.

Midwives felt that once violence cases were identified, dealing with those cases could be an issue to grapple with as they had no faith in the criminal justice system. The perceived reluctance of the courts to deal with reported cases was an obstacle to responding to violence. The midwife at Vurinda clinic stated that she had "never seen courts taking these issues seriously, so no seriousness in these violence cases'. This perception influenced their thinking that abuse cases would not be fairly and satisfactorily dealt with.

\section{'And this organisation is no longer coming to screen'}

A midwife at Chineka clinic described how a non-governmental organisation working at their clinic helped their clients who were abused and how the health system never tapped this opportunity for the midwives to refer or assist abused women after the non- governmental organisation left. She reported:

'We used to have this organization. ... They were after these pregnancy issues. They could see them [pregnant women] from the initial bookings and talking to them and giving them information about ... any problems at home with the husband. They were here to assist them. It's the one that looked after that. As for us we don't ... And this organisation is no longer coming. I don't know what happened ... They used to come during the initial visit and ... subsequent visits ... They would also hold interviews with mothers in the post- delivery section especially the unbooked mothers; they would want to know the reasons why they were not booked. Some mothers would say I didn't have the money because my husband was refusing to give me the money. They would ask if he was bringing her food.... The organisation would pay a certain amount and the other amount would be paid by the City of Harare [health department] ... (Fadzai, midwife, Chineka Clinic)

In this case, the midwife lamented over an opportunity lost in which they could have learned from the non-governmental organisation they worked with to screen and deal with IPV cases. She also reported that she often recommended to the city health authorities to introduce a screening programme, a recommendation that was never considered:

'So we have talked about it [IPV screening] so that it is included in our planning. ... the days when that organisation came ... we left those issues to them because they were trained and they had a trained nurse and midwife. So to us as Facility F ... we now see that it's [IPV] not being talked about ... But I think we need to include that in our programme ... Because we believe that some of them have got problems ... 'We were never put onto that programme... though we just talk about the problems we face at ANC when we submit our reports' (Fadzai, midwife, Chineka Clinic). 
An opportunity to integrate screening into the maternity care programme was lost. The midwife explained how the absence of the programme prevented them from assisting a woman:

'Like this person I still remember she was saying her husband has another wife but she is the first wife [and was being neglected]. .. . The husband was not giving her money to come and register. In the end she had to go and seek money from her brother in Mazowe. That's when she came to register but we used to see [at the clinic] that she was pregnant but not booked. And we were now in a dilemma as to what we were going to do about her. To someone who opens up that she does not have the money the Council [Health Department] says that she can pay half and then pay the rest after delivery. [She then travelled a long distance by bus and foot to get some money whilst she was nine months pregnant]. That's when she came to register and to make matters worse she had some complications and we transferred her to Mbuya Nehanda [tertiary hospital]' (Fadzai, midwife, Chineka Clinic)

Booking pregnancies in antenatal care involved paying a registration fee of US $\$ 50$, non- payment of which would deprive a pregnant woman of access to antenatal care services. In this case, the nurses watched her situation helplessly, recognizing that she was not booked due to financial problems. Further inquiry showed that nurses suspected that the situation at home and the travelling when she had problems walking contributed to complications leading her to be referred to a tertiary hospital. This case also shows how policy and structural factors together with gender-based violence create women's vulnerability.

\section{Culture, confidentiality and the law}

On probing, midwives reported a number of cultural factors influencing responses to IPV. They described women's non-disclosure of domestic issues as a 'culture of silence'. They perceived this to be one of the major challenges in responding to IPV at the clinic. A midwife said: Aah I don't know whether we will get that many women who will be open about that. Most women are secretive about domestic violence." (Bridget, midwife, Nekanda Clinic). Another midwife reported similar difficulties and recognised the health implications of non-reporting saying:

'They don't open up. ... Because like now she could have been admitted [she referred to a woman who miscarried but after investigations it was discovered that she was experiencing domestic violence]. You sit down with her and try to talk ... they don't open up but you can clearly see that there is a problem' (Fadzai, midwife, Chineka Clinic).

However, beyond - and compounding- a culture of silence regarding domestic violence, an important reason for women's reluctance to report appears to be related to midwives not being trusted with the information. Women suspected that nurses do not keep their domestic stories confidential and fear of the repercussions from their partners should they discover that the women reported the abuse was a barrier. Women also feared that such issues could spread into the community since a number of midwives lived in the same community with them. Midwives added that women 
feared that if they disclosed violence at the clinic, their husbands would end up in jail, thereby risking their family life and economic livelihoods. For example, midwives reported:

'There is also a tendency of women protecting their husbands because of this Domestic Violence Act. The moment you try to talk and ask them they think you want to drag their husbands to the police' (Diana, midwife, Madzive clinic).

It was also noted that violent experiences that happened between a woman and her partner were regarded as domestic and could not be discussed with other people outside. One midwife mentioned, 'They may have this negative attitude of saying I cannot discuss my family issues with the nurse' (Fadzai, midwife at Chineka Clinic).

Participants in the FGD and our observations revealed that there was limited infrastructure to facilitate privacy during history taking at five of the six clinics studied. It was observed during participant observation and in FGD women explained the lack of a private space during interactions with midwives. All services took place in close proximity of other pregnant women seated on benches waiting their turn. In general, most of the activities of the antenatal care visits took place in a foyer/hall or entrance, which is also used by visitors and staff of the maternity ward and clinic offices. Any disclosure of personal or domestic information would be heard by others on queue.

\section{Discussion}

The study presents a situational analysis around midwives and their thoughts of and experiences with identifying and responding to IPV against women during pregnancy, as well as the experiences of their clients. The study reveals the complexity and difficulty in responding to IPV in antenatal and postnatal care in a resource-limited setting. It showed that responding to IPV in antenatal settings is difficult for both health-sector related reasons and midwives' own embeddedness in a patriarchal culture which normalises IPV. Challenges include midwives' lack of specific education and training; high workloads and time pressures; the health system's lack of infrastructure, privacy, guiding policy, support, staff and other resources; midwives' own beliefs and previous experiences; women's non-disclosure of domestic issues; and cultural taboos. These multiple- layered complexities created barriers at different levels and any intervention would need to address these barriers in an integrated and comprehensive way. However, the potential impact of sensitisation and informal training could be seen from Facility $\mathrm{F}$ staff, who acquired some knowledge of responding to IPV by observing and interacting with trained staff of the non-governmental organisation that ran a screening programme at their clinic. This intervention programme, although directed at the pregnant women also had an unintended positive effect on the staff.

Our findings echo earlier findings on the lack of gender-based violence training and education among midwives (Bacchus, Mezey, and Bewley 2002; Erickson, Hill, and Siegel 2001; O'Reilly 2007; Stenson, Sidenvall, and Heimer 2005; Waalen et al. 2000), reflecting health policy silence regarding gender-based violence. This study 
suggests that initiating screening as suggested in developed settings would be challenging in resource-limited settings. Research in South Africa demonstrated that screening all women seeking care is expensive, while responding to specific cues could help address the problem with less human and financial resources (Joyner and Mash 2012). The context of staff shortages makes the additional task of screening, especially for a non-biological problem, much more difficult to accept, hence the need to develop practices that tap into nurses' clinical training and practices and also those that respond to the needs of patients. In busy public health settings, it is possible that not all women need be screened but that those at high risk be identified. It is encouraging that midwives themselves felt that training of nurses could play a role, as some were sensitised by being exposed to services that support IPV survivors. Also, whilst midwives were not trained in gender-based violence, some were able to assist or refer abused women after recognising cues.

The midwives' opportunities to address IPV in health promotion lessons were, however, squandered as pregnant women in the study reported being advised to submit to sexual violence perpetrated by their partners. The opportunity could have been utilised positively to empower pregnant women to negotiate safer sex if the midwives believed it to be the right thing and also if they knew how to do it. Midwives' main concern was HIV prevention by preventing men from having multiple concurrent sexual relationships, which could create HIV risk for women. However, this was done without any consideration of its impact on sexual violence. These and other data from our study suggest that midwives perceived sexual violence as a normal phenomenon and that the link between sexual violence and HIV-risk among pregnant women needs to be better understood in the context of improved antenatal care. The opportunity of group health promotion lessons with each antenatal visit could have additional benefits if midwives were trained to offer IPV group awareness and counselling.

The study revealed health system shortcomings regarding responding to and assisting abused women. Firstly, we find a disjuncture between health system policy and health workers' operations. Whilst midwives recommended to health managers the need to implement a programme that would help them to identify and assist abused women, the latter did not see it as a priority. The fact that pregnant women without money could not afford to access maternity health services and the inability of nurses to at least bring those not able to pay to the attention of facility management for waiver of fees (as per the local health system policy), reveals significant health system failure. It also meant that pregnant women missed the opportunity of ante natal care which has a huge impact on maternal and child health since those who failed to pay antenatal care fees only accessed care when in labour or delivered at home. Secondly, the non-sustainability of new health interventions that run parallel to the existing system in antenatal care deserves attention.

The non-governmental organisation that operated at Facility F was a good example of an unsustainable parallel intervention. There is need to integrate such interventions in existing health care programmes for a sustained service. 
Despite evidence showing patients' positive attitude towards domestic violence screening (Gielen et al. 2000; Ramsay et al. 2002; Webster, Stratigos, and Grimes 2001) health workers in our study reported women's perceived non-disclosure of violence as a major challenge. Whilst providers cited a culture of silence on the part of pregnant women, the latter reported unavailability of private space to discuss their personal and domestic problems with their providers and this was also illustrated by the fact that some women would follow a nurse at home or church to discuss their problems. Structural and infrastructural issues in the health system must be addressed to enable responding to IPV in antenatal care. The fact that pregnant women in the study had low incomes, many of them unemployed and dependent on their partners, could also explain the perceived fear to disclose their abuse experiences. For women, disclosing their abuse experiences implied that their partners could be jailed thereby ruining their economic livelihoods. They also feared further abuse after they reported their partners as abusive. The perception of some respondents that other government departments were not taking domestic violence seriously was corroborated by Chirawu (2006), who argued that there has not been a single case of IPV brought to the courts for prosecution by 2006 since sexual offenses law was enacted in 2001, despite high prevalence of IPV in Zimbabwe.

Midwives seemed to emphasise that reporting violence results in progressing to court as the only outcome, yet attrition studies (Jewkes and Abrahams 2002) show that most women do not want to progress to the court system. Midwives did not recognise that they could play a significant role just by listening to women narrating their problems. Hindin (2006) found that there was need to enter into a trusting relationship with the women before screening. The need for appropriate and culturally relevant ways of asking the screening questions has been reported in Japan (Kataoka et al. 2004) and in the USA (Hindin 2006). The midwives in our study raised difficulties asking questions in their vernacular, which may suggest that any screening tool designed should be culturally relevant.

To the best of our knowledge, this is the first study in Zimbabwe to look at perceptions and experiences of midwives and pregnant/nursing mothers regarding responding to pregnant women for IPV. Further research is needed to understand the non-health system obstacles to IPV responses such as the views and experiences of the police, courts and organisations that provide care to abused women. The study could have also looked at women's experiences with midwives in detail to understand better the provider-client interface.

\section{Conclusion}

The study identified obstacles to responding to IPV in antenatal care at the level of the health system, midwives and pregnant and nursing women and the role of a patriarchal culture that subjugates the needs of women and that in the clinical setting, reproduces hierarchies between nurses and patients, which further silence women experiencing IPV. Lack of education and skills to screen for violence, the health system's limited human resources capacity and its failure to promote screening, and role conflicts reflecting a deep ambivalence over whether IPV is a health problem, a 
social problem or simply 'normal' were identified as the major obstacles to responding to IPV. The study highlighted the importance of antenatal and postnatal care sessions as opportunities to identify and respond to IPV issues. Training and supporting nurses to pay attention to cues in responding, in a sensitive caring way, to IPV may be a more feasible first step than universal screening. Institutional reform that will lead to training midwives and integrating IPV responses in antenatal care services could help in addressing IPV in antenatal care in culturally appropriate and sensitive ways.

\section{Acknowledgements}

The first author received funding for his doctoral studies, towards which this paper contributes, from Flemish Inter-University Cooperation (VLIR-UOS) at the University of the Western Cape, whilst fieldwork for this paper was supported by the University of the Western Cape's Faculty Research grant managed by Christina Zarowsky. The first author attended writing workshops sponsored by the African Doctoral Dissertation Fellowship (ADDRF), during which this paper was prepared for publication. We are grateful to the research assistant, Halleluah Chirisa and to Godfrey Shamu who also assisted with transcribing and translating interviews. 


\section{References}

Audi, C.A., A.M. Correa, R. Latorre Mdo, and S.M. Santiago. 2008. "The Association Between Domestic Violence During Pregnancy and Low Birth Weight or Prematurity." Jornal de Pediatria 84 (1): 60-7.

Bacchus, L., G. Mezey, and S. Bewley. 2002. "Women's Perceptions and Experiences of Routine Enquiry for Domestic Violence in a Maternity Service." BJOG: An International Journal of Obstetrics and Gynaecology 109 (1): 9-16.

Bacchus, L., G. Mezey, S. Bewley, and A. Haworth. 2004. "Prevalence of Domestic Violence When Midwives Routinely Enquire in Pregnancy." BJOG: An International Journal of Obstetrics and Gynaecology 111 (5): 441-5.

Bacchus, L.J., S. Bewley, C. Torres Vitolas, G. Aston, P. Jordan, and S.F. Murray. 2010. "Evaluation of a Domestic Violence Intervention in the Maternity and Sexual Health Services of a UK Hospital." Reproductive Health Matters 18 (1): 147-57.

Chirawu, S. 2006. "Till Death do us Part: Marriage, HIV/AIDS and the Law in Zimbabwe." Cardozo Journal of Law and Gender 13 (36): 23-50.

CSO, and Macro. 2007. Zimbabwe Demographic and Health Survey 2005 - 2006. Calverton, Maryland: CSO and Macro International Inc.

Erickson, M.J., T.D. Hill, and R.M. Siegel. 2001. "Barriers to Domestic Violence Screening in the Pediatric Setting." Pediatrics 108 (1): 98-102.

Gielen, A.C., P.J. O’Campo, J.C. Campbell, J. Schollenberger, A.B. Woods, A.S. Jones, J.A. Dienemann, J. Kub, and E.C. Wynne. 2000. "Women's Opinions About Domestic Violence Screening and Mandatory Reporting." American Journal of Preventive Medicine 19 (4): 279-85.

Heise, L., M. Ellsberg, and M. Gottmoeller. 2002. "A Global Overview of Gender-Based Violence.” International Journal of Gynecology and Obstetrics 78: S5-S14.

Hindin, P.K. 2006. "Intimate Partner Violence Screening Practices of Certified NurseMidwives." Journal of Midwifery and Women's Health 51 (3): 216-21.

Jewkes, R., and N. Abrahams. 2002. "The Epidemiology of Rape and Sexual Coercion in South Africa: An Overview." Social Science \& Medicine 55: 1231-44.

Joyner, K., and R. Mash. 2012. "Recognizing Intimate Partner Violence in Primary Care: Western Cape, South Africa." PLoS One 7 (1): 5.

Kataoka, Y., Y. Yaju, H. Eto, N. Matsumoto, and S. Horiuchi. 2004. "Screening of Domestic Violence Against Women in the Perinatal Setting: A Systematic Review." Japan Journal of Nursing Science 1 (2): 77-86.

O’Campo, P., M. Kirst, C. Tsamis, C. Chambers, and F. Ahmad. 2011. "Implementing Successful Intimate Partner Violence Screening Programs in Health Care Settings: Evidence Generated from a Realist-Informed Systematic Review.” Social Science and Medicine 72 (6): 855-66.

O’Reilly, R. 2007. "Domestic Violence Against Women in Their Childbearing Years: A Review of the Literature." Contemporary Nurse 25 (1-2): 13-21.

Ramsay, J., J. Richardson, Y.H. Carter, L.L. Davidson, and G. Feder. 2002. "Should Health Professionals Screen Women for Domestic Violence? Systematic Review.” British Medical Journal 325 (7359): 314.

Roelens, K., H. Verstraelen, K. Van Egmond, and M. Temmerman. 2006. "A Knowledge, Attitudes, and Practice Survey Among Obstetrician-Gynaecologists on Intimate Partner Violence in Flanders, Belgium.” BMC Public Health 6: 238. 
Shamu, S., N. Abrahams, M. Temmerman, A. Musekiwa, and C. Zarowsky. 2011. "A Systematic Review of African Studies on Intimate Partner Violence Against Pregnant Women: Prevalence and Risk Factors." PloS one 6 (3): e1759.

Silverman, J.G., M.R. Decker, E. Reed, and A. Raj. 2006. "Intimate Partner Violence Victimization Prior to and During Pregnancy Among Women Residing in 26 U.S. States: Associations With Maternal and Neonatal Health." American Journal of Obstetetrics and Gynecology 195 (1): 140-8.

Stenson, K., B. Sidenvall, and G. Heimer. 2005. "Midwives' Experiences of Routine Antenatal Questioning Relating to Men's Violence Against Women.” Midwifery 21 (4): 311-21.

Taillieu, T.L., and D.A. Brownridge. 2010. "Violence Against Pregnant Women: Prevalence, Patterns, Risk Factors, Theories, and Directions for Future Research." Aggression and Violent Behavior 15 (1): 14-35.

Undie, C., C. Maternowska, M. Mak’anyengo, H. Birungi, J. Keesbury, and I. Askew. 2012. "Routine Screening for Intimate Partner Violence in Public Health Care Settings in Kenya: An Assessment of Acceptability.” In APHIA II OR Project in Kenya. Nairobi, Kenya: Population Council.

Waalen, J., M.M. Goodwin, A.M. Spitz, R. Petersen, and L.E. Saltzman. 2000. "Screening for Intimate Partner Violence by Health Care Providers. Barriers and Interventions." American Journal of Preventive Medicine 19 (4): 230 - 7.

Webster, J., S.M. Stratigos, and K.M. Grimes. 2001. "Women's Responses to Screening for Domestic Violence in a Health-Care Setting." Midwifery 17 (4): 289-94.

WHO. 2005. WHO Multi-County Study on Women's Health and Domestic Violence Against Women: Initial Results on Prevalence, Health Outcomes and Women's Responses. Geneva: WHO. 